\title{
FACTORES DE RIESGO CARDIOVASCULAR Y PERFIL APOLIPOPROTÉICO EN UN GRUPO DE ADULTOS ATENDIDOS EN UN CENTRO PUBLICO DE SALUD DEL ESTADO CARABOBO, VENEZUELA
}

\author{
Nelina Ruiz',2,a, Valerie Castillo, ${ }^{3, b}$, Francys Colina ${ }^{3, b}$, Milagros Espinoza ${ }^{3,4, c}$, \\ Ulises Leal ${ }^{5, \mathrm{~d}}$, Julio César Gonzalez ${ }^{4, \mathrm{e}}$.
}

\begin{abstract}
RESUMEN
Objetivos. Comparar los niveles séricos de las apolipoproteínas A-I y B así como las relaciones Apo B/Apo A-I y HDL colesterol/Apo A-I según edad, sexo y factores de riesgo cardiovascular en individuos atendidos en un centro público de salud venezolano. Materiales y métodos. Se determinó la presión arterial, la circunferencia de cintura (CC), el perfil lipídico y las apolipoproteínas A-I y B en 221 individuos (44,0 15,5 años) de ambos sexos; también se calculó el índice de masa corporal (IMC) a partir del peso y la talla y se estableció hábito al tabaco, la ingesta de bebidas alcohólicas y el patrón de su consumo. Resultados. El 27,5\% presentó concentraciones bajas de Apo A-I, 45,2\% Apo B elevada y 60,6\% relación Apo B/Apo A-I alta. Los niveles séricos de las apolipoproteínas y la relación Apo B/Apo A-I no variaron con la edad o sexo, mientras que la relación HDL colesterol/Apo A-I disminuyó al elevarse la edad. Los individuos obesos, fumadores, hipertensos, hipercolesterolémicos, hipertrigliceridémicos o con HDL colesterol bajo mostraron cifras más elevadas de Apo B y Apo B/Apo A-I. La relación HDL colesterol/Apo A-I disminuyó con la edad, el nivel de habito al tabaco y el aumento de LDL-C y triglicéridos. El consumo de tres o más bebidas alcohólicas/día se asoció con disminución de Apo B. Conclusiones. Se demostró alta prevalencia de perfil apolipoprotéico alterado, lo cual se asoció con los principales factores de riesgo cardiovascular. Los resultados del estudio apoyan la inclusión de las apolipoproteínas evaluadas en las determinaciones de laboratorio realizadas en los centros públicos de atención de salud venezolanos.
\end{abstract}

Palabras clave: Apolipoproteína A-l; Apolipoproteínas B; Enfermedades cardiovasculares; Obesidad; Hipertensión; Tabaquismo; Bebidas alcohólicas. (fuente: DeCS BIREME).

\section{CARDIOVASCULAR RISK FACTORS AND APOLIPOPROTEIC PROFILE IN A GROUP OF ADULTS TREATED IN A PUBLIC HEALTH CENTER IN CARABOBO STATE, VENEZUELA}

\begin{abstract}
Objectives. To compare serum levels of apolipoproteins A-I and B as well as Apo B/Apo A-I and HDL cholesterol/Apo A-I ratios by age, gender and cardiovascular risk factors in individuals treated at a Venezuelan public health center. Materials and methods. We determined in 221 individuals (44.0 \pm 15.5 years) of both genders blood pressure, waist circumference (WC), lipid profile and apolipoproteins A-I and B; body mass index (BMI) was calculated from weight and height; smoking habit, alcohol intake and consumption pattern were established. Results. $27.5 \%$ of individuals had low levels of Apo A-I, 45.2\% high Apo B and 60.6\% high Apo B/Apo A-I ratio. Serum levels of apolipoproteins and Apo B/Apo A-I ratio did not vary with age or gender, while the ratio HDL cholesterol/Apo A-I decreased with the age. Obese individuals, smokers, hypertensive, hypercholesterolemics, hypertriglyceridemics or with low HDL cholesterol showed higher Apo B and Apo B/Apo A-I ratio. Older individuals, smokers or individuals with increased LDL cholesterol and triglycerides showed lower HDL cholesterol/Apo A-I ratio. Consumption of three or more alcoholic drinks/day was associated with decreased Apo B. Conclusions. These results show high prevalence of altered apolipoprotein profile, which is associated with major cardiovascular risk factors. The results support the inclusion of the evaluated apolipoproteins in laboratory determinations made in public health centers in Venezuela.
\end{abstract}

Key words: Apolipoprotein A-I; Apolipoproteins B; Cardiovascular diseases; Obesity; Hypertension; Smoking; Alcoholic beverages. (source: MeSH NLM).

\footnotetext{
Departamento de Morfofisiopatología. Escuela de Bioanálisis Sede Carabobo, Facultad de Ciencias de la Salud, Universidad de Carabobo. Valencia, Venezuela.

2 Instituto de Investigaciones en Nutrición (INVESNUT), Facultad de Ciencias de la Salud, Universidad de Carabobo. Valencia, Venezuela.

3 Departamento de Investigación y Desarrollo Profesional. Escuela de Bioanálisis Sede Carabobo. Universidad de Carabobo. Valencia, Venezuela.

4 Centro de Investigaciones Médicas y Biotecnológicas de la Universidad de Carabobo (CIMBUC), Facultad de Ciencias de la Salud. Valencia, Venezuela.

5 Unidad de Atención Médico Integral de la Universidad de Carabobo (UAMI). Valencia, Venezuela.

a Doctor en Ciencias Fisiológicas; ${ }^{b}$ Licenciado en Bioanálisis; ${ }^{\circ}$ Magister en Gerencia y Tecnología de Información. ${ }^{d}$ Especialista en Medicina Interna; e Doctor en Química Clínica.
}

Recibido: 04-02-11 Aprobado: 01-06-11 


\section{INTRODUCCIÓN}

Las apolipoproteínas son constituyentes estructurales imprescindibles de las partículas lipoprotéicas, que se diferencian no solo por su composición en aminoácidos sino por las funciones que cumplen (1). La apolipoproteína A-I (Apo A-I) es la principal proteína estructural de las lipoproteínas de alta densidad (HDL) y está directamente involucrada en el transporte reverso de colesterol (2). En contraste, la apolipoproteína B (Apo B) es la proteína estructural de las lipoproteínas aterogénicas (lipoproteína de muy baja densidad o VLDL, lipoproteína de densidad intermedia o IDL y de la lipoproteína de baja densidad o LDL) que transportan colesterol hacia los tejidos periféricos; cada partícula de estas lipoproteínas contiene una molécula de Apo B, por lo que el nivel en sangre de esta apolipoproteína se corresponde con el número total de partículas aterogénicas del plasma (1).

Hallazgos de diferentes estudios han apuntado que las concentraciones séricas de Apo B y Apo A-I y la relación Apo B/Apo A-I predicen mejor el riesgo cardiovascular que otros indicadores rutinarios del perfil lipídico ${ }^{(3,4)}$. Por otra parte, se ha señalado que la relación HDL coleste$\mathrm{rol} / \mathrm{Apo} \mathrm{A}-\mathrm{I}$ constituye un buen indicador de la eficiencia de la vía de transporte del colesterol desde los tejidos hacia el hígado ${ }^{(5)}$. No obstante, no se disponen de datos amplios sobre los niveles séricos de Apo A-I y de Apo $B$, ni de las relaciones Apo B/Apo A-I (rApo B/Apo A-I) y HDL colesterol/Apo A-I (rHDLc/Apo A-I) entre los venezolanos, a pesar de que múltiples estudios ${ }^{(6-8)}$, desarrollados en diferentes zonas del país, han informado una importante prevalencia de alteraciones cardiometabólicas asociadas con elevado riesgo cardiovascular, entre las que destacan las alteraciones del perfil lipídico. El propósito de esta investigación fue comparar los niveles séricos de las apolipoproteínas y las relaciones mencionadas según edad, sexo y factores de riesgo cardiovascular en un grupo de individuos aparentemente sanos, atendidos en un centro público de salud localizado en el Estado Carabobo, Venezuela.

\section{MATERIALES Y MÉTODOS}

Estudio descriptivo-transversal, de muestra no probabilística e intencional, llevado a cabo en 221 individuos de ambos sexos, que asistieron a la consulta especializada de un centro público de salud ubicado en el Municipio San Diego, ciudad de Valencia, Estado Carabobo, Venezuela entre enero y julio de 2010. En el municipio mencionado residen individuos de diferentes estratos socioeconómicos, pero fundamentalmente acuden al centro público de salud, aquellos ubicados en los estratos medios y bajos, según los registros del centro. Los criterios de inclusión fueron: edad $\geq 18$ años, aparentemente sanos, sin antecedentes médicos personales de enfermedad cardiovascular (infarto agudo al miocardio, enfermedad cardíaca isquémica, insuficiencia cardíaca, accidente cerebro-vascular, arteriopatías periféricas y trombosis venosas profundas), enfermedad renal, diabetes mellitus u otra enfermedad endocrina, hepatopatías y cáncer. Se excluyeron gestantes y pacientes bajo terapia hipolipemiante u hormonal. Se cumplieron todos los acuerdos de la Declaración de Helsinki, pues se obtuvo el consentimiento informado firmado de los individuos evaluados. El protocolo de estudio fue aprobado por la Comisión de Ética y Dirección del centro sanitario.

Mediante encuesta se obtuvieron datos personales y sociodemográficos, antecedentes personales y familiares en primer grado de consanguinidad de diabetes mellitus (DM), hipertensión arterial (HTA), enfermedad cardíaca isquémica $(\mathrm{ECl})$, accidente cerebrovascular $(\mathrm{ACV})$, otros antecedentes médicos personales, hábito al tabaco, consumo de bebidas alcohólicas y tratamiento farmacológico. Los procedimientos empleados para medir peso, talla, circunferencia de cintura (CC) y presión arterial se han descrito previamente ${ }^{(7)}$. Se calculó el índice de masa corporal o $\mathrm{IMC}\left(\mathrm{kg} / \mathrm{m}^{2}\right)$.

Previo ayuno de $12-14$ horas, se extrajeron $10 \mathrm{~mL}$ de sangre venosa en el pliegue del codo. El mismo día se determinaron los niveles de colesterol total, triglicéridos, colesterol-HDL (HDLC) y colesterol-LDL (LDLC) mediante métodos enzimáticos-colorimétricos; los dos últimos previa precipitación. Otra alícuota de suero se empleó para determinar Apo B y Apo A-I mediante métodos inmunoturbidimétricos. Todas las mediciones se realizaron de modo manual en un espectrofotómetro Stat Fax Omega IV, empleando reactivos para perfil lipídico de la casa comercial Bioscience (Caracas, Venezuela) y de LabKit (Barcelona, España) para las apolipoproteínas. Se calcularon las relaciones rApo B/Apo A-I y rHDLc/Apo A-I. Valores de Apo A-I menores de $1,07 \mathrm{mg} / \mathrm{dL}$ y $1,22 \mathrm{mg} /$ $\mathrm{dL}$, en hombres y mujeres respectivamente, se consideraron bajos; valores de Apo $B$ mayores de $1,2 \mathrm{mg} / \mathrm{dL}$ y de rApo B/Apo A-I por encima de 0,8 en hombres y de 0,7 en mujeres fueron definidos elevados ${ }^{(9)}$.

Los factores de riesgo cardiovascular considerados en este estudio fueron: obesidad según IMC y $\mathrm{CC}$, hábito tabáquico, HTA, consumo de alcohol y dislipidemias. Se definió como no fumador aquel participante que nunca había fumado o con más de cinco años de haber dejado el tabaco y como fumador aquel que fumaba al momento del estudio o que había abandonado este hábito dentro de los cinco años previos a la evaluación ${ }^{(10)}$. Para establecer sobrepeso/obesidad, HTA y alteraciones del perfil lipídico se emplearon criterios aceptados internacionalmente ${ }^{(11-13)}$. 
El patrón de consumo de alcohol se evaluó de acuerdo con la Prueba de Identificación de Desórdenes del Uso del Alcohol (The Alcohol Use Disorders Identification Test o AUDIT) ${ }^{(14)}$, la cual considera tres dimensiones: frecuencia del consumo, cantidad usualmente bebida y frecuencia con la cual el individuo bebe seis o más bebidas/ocasión. La frecuencia del consumo se agrupó así: nunca, 1 a 3 veces/mes o menos, 1 o más días/ semana. La cantidad bebida ingerida se agrupó como sigue: 1, 2 a 4, 5 a 6 bebidas/día que toma alcohol. La frecuencia de ingesta de seis o más bebidas en una ocasión se agrupó: nunca, menos que mensualmente, mensualmente (1 a 3 veces/mes), semanalmente (1 vez/semana), diariamente o casi diariamente (más de 1 vez/semana). El período de tiempo con base al cual se revisó el consumo fue los últimos 12 meses previos a la evaluación.

Se calcularon estadísticos descriptivos de tendencia central y dispersión, frecuencias absolutas y relativas. Se verificó la distribución normal de las variables estudiadas mediante test de Kolmogorov Smirnov. Se empleó prueba de t-student para comparar los niveles séricos promedios de las apolipoproteínas y rApo B/Apo A-I según obesidad abdominal, hábito tabáquico, HTA, consumo de alcohol y alteraciones del perfil lipídico, mientras que se utilizó el análisis de ANOVA y la prueba post hoc de Bonferroni para comparar según IMC y patrón de consumo de alcohol. Se aplicaron las pruebas no paramétricas correspondientes a rHDLc/Apo A-I por no seguir la distribución normal. Se utilizó prueba de chi-cuadrado para comparar la frecuencia de perfil apolipoprotéico alterado según edad y factores de riesgo cardiovascular estudiados. Se empleó el paquete estadístico PASW Statistics Multilenguaje versión 18.0 y un nivel de significancia de $p<0,05$.

\section{RESULTADOS}

Se estudiaron 221 individuos con edad promedio de

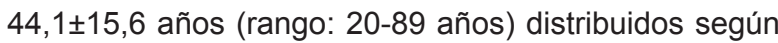
sexo: 104 hombres y 117 mujeres; existió diferencia significativa en edad a favor de las mujeres $(31,9 \pm 10,5$ frente a 47,0 $\pm 14,6$ años; $p<0,001)$. La frecuencia de antecedentes familiares de HTA, ECl, ACV y DM en el grupo estudiado fue de 65,$4 ; 30,9 ; 33,6$ y $44,1 \%$ respectivamente. En la Tabla 1 se observan las características generales de la muestra. Los factores de riesgo cardiovascular estudiados mostraron la siguiente frecuencia: $68,5 \%$ presentó IMC elevado (sobrepeso $47,3 \%$ y obesidad 21,2\%), 47,3\% mostró obesidad abdominal, 29,7\% hipercolesterolemia, 58,6\% HDLc baja, 29,7\% LDLc elevada, $44,1 \%$ triglicéridos elevados y $21,6 \%$ fueron fumadores. Las frecuencias de hipercolesterolemia y de triglicéridos elevados no se asociaron con la edad, mientras que las del resto de los factores de riesgo cardiovascular estudiados fueron significativamente mayores en los individuos de más edad.

Entre los individuos que informaron consumo de bebidas alcohólicas $(n=58 ; 27,1 \%)$, la mayoría lo hizo con frecuencia menor a una vez a la semana $(79,3 \%)$ y solamente 3,4\% $(n=2)$ ingirió bebidas alcohólicas tres o más días a la semana; casi dos tercios de la muestra (60,3\%) reportó que bebía tres o más bebidas/día y sólo15 individuos $(25,9 \%)$ informaron que consumían seis o más bebidas alcohólicas/ocasión con frecuencia semanal o diaria.

En 61 individuos $(27,5 \%)$ se encontró valores bajos de Apo A-I y en 99 (45,2\%) concentraciones elevadas de Apo B. La rApo B/Apo A-I se encontró elevada en el $60,6 \%$ de los casos. Al comparar por sexos, la rApo B/ Apo A-I promedio fue significativamente mayor entre las mujeres ( 1,06 vs. 0,$95 ; p=0,01)$, las cuales también presentaron frecuencia superior de Apo A-I baja y rApo B/ Apo A-I elevada $(p<0,001)$.

Los niveles séricos de las apolipoproteínas y la rApo B/ Apo A-I no variaron significativamente según edad en el grupo total; resultado similar se obtuvo cuando se realizó el mismo análisis diferenciado por sexo (Tabla 2). En el grupo total, la rHDLc/Apo A-I fue significativamente menor en el grupo de participantes de mayor edad en relación al grupo de adultos jóvenes y de mediana edad, este mismo comportamiento se halló cuando se anali-

Tabla 1. Características basales de la población estudiada*.

\begin{tabular}{lcccc}
\hline \multicolumn{1}{c}{ Variable } & Mínimo & Máximo & Media & DE \\
\hline Edad (años) & 20,0 & 89,0 & 44,0 & 15,5 \\
Peso $(\mathrm{kg})$ & 45,0 & 124,0 & 69,3 & 11,5 \\
Talla $(\mathrm{m})$ & 1,50 & 1,90 & 1,61 & 0,1 \\
IMC $\left(\mathrm{kg} / \mathrm{m}^{2}\right)$ & 18,5 & 47,8 & 27,4 & 4,4 \\
CC $(\mathrm{mm})$ & 74,0 & 145,0 & 94,5 & 9,8 \\
PAS $(\mathrm{mmHg})$ & 90,0 & 190,0 & 121,6 & 14,2 \\
PAD $(\mathrm{mmHg})$ & 60,0 & 120,0 & 83,4 & 10,6 \\
CT $(\mathrm{mg} / \mathrm{dL})$ & 95,0 & 433,0 & 188,3 & 41,9 \\
HDLc $(\mathrm{mg} / \mathrm{dL})$ & 23,0 & 82,0 & 42,7 & 8,0 \\
LDLc $(\mathrm{mg} / \mathrm{dL})$ & 35,0 & 344,0 & 115,6 & 41,2 \\
TG $(\mathrm{mg} / \mathrm{dL})$ & 38,0 & 754,0 & 158,1 & 80,5 \\
Apo A-I $(\mathrm{mg} / \mathrm{dL})$ & 74,2 & 194,0 & 126,6 & 18,8 \\
Apo B $(\mathrm{mg} / \mathrm{dL})$ & 41,0 & 217,0 & 124,3 & 30,0 \\
rApo B/Apo A-I & 0,29 & 2,36 & 1,01 & 0,33 \\
rHDLc/Apo A-I & 0,17 & 0,72 & 0,34 & 0,08 \\
\hline
\end{tabular}

${ }^{*} \mathrm{n}=221$. IMC: índice de masa corporal; CC: circunferencia de cintura; PAS: presión arterial sistólica; PAD: presión arterial diastólica; CT: colesterol total; HDLc: colesterol unido a la lipoproteína de alta densidad; LDLc: colesterol unido a la lipoproteína de baja densidad; TG: triglicéridos; Apo: apolipoproteína. DE: desviación estándar. 


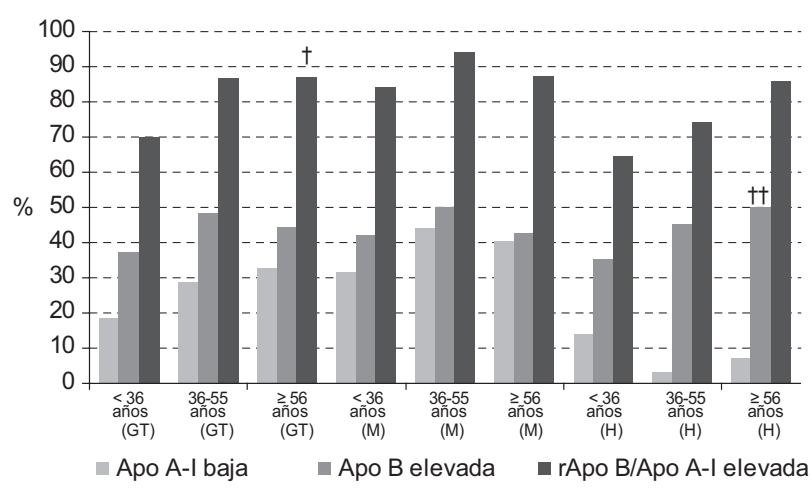

Figura 1. Frecuencia de valores alterados de Apolipoproteínas A-I, B y rApo B/Apo A-I según edad y género.

${ }^{*} n=221 .{ }^{\dagger} p<0,05 ;{ }^{\dagger t} p<0,01$. GT: grupo total; M: mujeres; H: hombres; Apo: apolipoproteína.

zaron los hombres por separado (Tabla 2). Cuando se analizó la frecuencia de valores alterados de las apolipoproteínas y de la relación $\mathrm{rApo} B / A p o A-I$ según edad y sexo, se observó en el grupo total que el porcentaje de individuos con rApo B/Apo A-I elevada aumento al avanzar la edad $(p<0,05)$ y que la prevalencia de Apo B elevada, fue significativamente mayor entre los hombres de más edad $(p<0,01)$ (Figura 1).

La Tabla 3 muestra las concentraciones séricas promedios de apolipoproteínas A-I y B, rApo B/Apo A-I y rHDLc/Apo A-I según factores de riesgo cardiovascular estudiados. Entre los individuos con exceso de peso corporal según IMC, con obesidad abdominal o con HTA se observaron valores promedios de Apo B y rApo B/ Apo A-I significativamente más elevados así como valores de Apo A-I más bajos. Los fumadores mostraron niveles más altos de Apo $\mathrm{B}$ y más bajos de la relación rHDLc/Apo A-I con respecto a los no fumadores. La frecuencia de Apo A-I baja fue significativamente más elevada entre los pacientes con exceso de peso $(p<0,01)$, con obesidad abdominal $(p<0,01)$ o con HTA $(p<0,01)$; la frecuencia de Apo B elevada fue significativamente

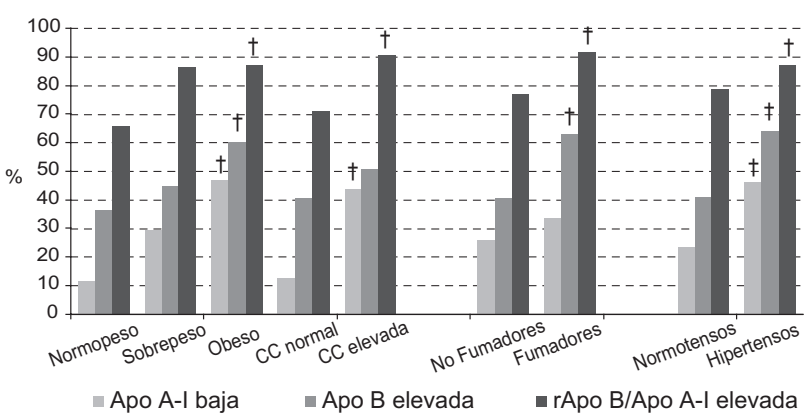

Figura 2. Frecuencia de valores alterados de Apolipoproteínas A-I, B y rApo B/Apo A-I según factores de riesgo cardiovascular. ${ }^{*} n=221 .+p<0,01$. CC: circunferencia de cintura; Apo: Apolipoproteína.

mayor entre los pacientes con exceso de peso $(p<0,01)$, que reportaron habito tabáquico $(p<0,01)$ o presentaron HTA $(p<0,01)$; la frecuencia de rHDLc/Apo A-I elevada fue asociada a todos los factores de riesgo evaluados (Figura 2).

Las apolipoproteínas A-I y B y las relaciones evaluadas no mostraron diferencias significativas según consumo de bebidas alcohólicas, frecuencia de ingesta o frecuencia de ingesta de seis o más bebidas en una ocasión (Tabla 4). Entre los individuos que informaron consumo de alcohol, aquellos que bebieron entre una y dos bebidas/ día mostraron niveles de Apo B significativamente más elevados en comparación a los que reportaron ingerir tres o más bebidas/día $(p<0,05)$. Debido al bajo número de participantes que reportaron ingesta de alcohol no fue estadísticamente posible asociar la proporción de casos de valores alterados de las apolipoproteínas estudiadas de acuerdo al patrón de consumo de bebidas alcohólicas.

Se observaron niveles significativamente mayores de Apo B y de rHDLc/Apo A-I entre los individuos estudiados con concentraciones séricas elevadas de colesterol total, LDLc y triglicéridos o HDLc baja (Tabla 5). Los individuos hipercolesterolémicos, con HDLc baja o hi-

Tabla 2. Niveles Séricos de Apolipoproteínas A-I y B, rApo B/Apo A-I y rHDLc/Apo A-I según género y edad*.

\begin{tabular}{lccc}
\hline \multicolumn{1}{c}{ Sexo y Edad } & Apo A-I $(\mathrm{mg} / \mathrm{dL})$ & Apo B $(\mathrm{mg} / \mathrm{dL})$ & rApo B/Apo A-I \\
\hline Grupo Total & & & \\
$<36$ años $(70)$ & $128,3 \pm 17,1$ & $118,4 \pm 26,6$ & $0,95 \pm 0,29$ \\
$36-55$ años (90) & $124,7 \pm 20,0$ & $125,7 \pm 31,5$ & $1,04 \pm 0,35$ \\
$\geq 56$ años (61) & $127,4 \pm 19,1$ & $129,0 \pm 30,9$ & $1,04 \pm 0,34$ \\
Mujeres & & & $0,35 \pm 0,07$ \\
$<36$ años (19) & $131,8 \pm 16,7$ & $121,1 \pm 25,0$ & $0.93 \pm 0,23$ \\
$36-55$ años (51) & $121,8 \pm 22,7$ & $130,4 \pm 31,5$ & $1,12 \pm 0,39$ \\
$\geq 56$ años (47) & $125,8 \pm 20,2$ & $128,3 \pm 32,9$ & $1,05 \pm 0,36$ \\
Hombres & & & $0,35 \pm 0,10$ \\
$<36$ años (52) & $127,0 \pm 17,1$ & $0,35 \pm 0,10$ \\
$36-55$ años (38) & $128,6 \pm 14,8$ & $117,4 \pm 27,3$ & $0,95 \pm 0,31$ \\
$\geq 56$ años (14) & $132,5 \pm 14,3$ & $119,2 \pm 30,7$ & $0,93 \pm 0,24$ \\
\hline
\end{tabular}

*n entre paréntesis. Resultados expresados como media aritmética \pm desviación estándar.

${ }^{\dagger} p<0,05$, grupo $\geq 56$ años vs grupo $36-55$ años o grupo $<36$ años.

Análisis de varianza y prueba de Bonferroni. 
Tabla 3. Niveles séricos de apolipoproteínas A-I y B, rApo B/Apo A-I y rHDLc/Apo A-I según factores de riesgo cardiovascular estudiados*.

\begin{tabular}{|c|c|c|c|c|}
\hline & Apo A-I (mg/dL) & Apo B $(\mathrm{mg} / \mathrm{dL})$ & rApo B/Apo A-I & rHDLc/Apo A-I \\
\hline \multicolumn{5}{|l|}{ IMC } \\
\hline Normopeso (70) & $136,4 \pm 19,0$ & $117,6 \pm 24,6$ & $0,89 \pm 0,25$ & $0,33 \pm 0,06$ \\
\hline Sobrepeso (104) & $124,2 \pm 15,3^{\text {t†a }}$ & $125,1 \pm 28,3$ & $1,03 \pm 0,31^{\text {t+a }}$ & $0,35 \pm 0,07$ \\
\hline Obesidad (47) & $117,3 \pm 19,6^{+\mathrm{tb}}$ & $132,6 \pm 35,1^{\text {tb }}$ & $1,15 \pm 0,41^{\dagger+b}$ & $0,35 \pm 0,11$ \\
\hline \multicolumn{5}{|c|}{ Obesidad abdominal según CC } \\
\hline Ausente (116) & $131,3 \pm 16,9$ & $120,0 \pm 26,7$ & $0,94 \pm 0,26$ & $0,34 \pm 0,06$ \\
\hline Presente (105) & $121,3 \pm 19,5^{t+c}$ & $129,1 \pm 31,1^{\text {tc }}$ & $1,10 \pm 0,38^{t+c}$ & $0,35 \pm 0,11$ \\
\hline \multicolumn{5}{|l|}{ Habito tabáquico } \\
\hline No Fumador (173) & $126,3 \pm 18,3$ & $121,2 \pm 28,8$ & $0,99 \pm 0,32$ & $0,35 \pm 0,08$ \\
\hline Fumador (48) & $127,1 \pm 20,7$ & $136,2 \pm 27,7^{\dagger t d}$ & $1,09 \pm 0,34$ & $0,31 \pm 0,07^{\dagger+d}$ \\
\hline \multicolumn{5}{|l|}{ Hipertensión arterial } \\
\hline Normotensos (182) & $127,9 \pm 18,5$ & $122,1 \pm 27,8$ & $0,98 \pm 0,30$ & $0,34 \pm 0,08$ \\
\hline Hipertensos (39) & $120,2 \pm 19,5^{\text {te }}$ & $134,4 \pm 33,2^{\text {te }}$ & $1,16 \pm 0,40^{\text {te }}$ & $0,36 \pm 0,09$ \\
\hline
\end{tabular}

"n entre paréntesis. Resultados expresados como media aritmética \pm desviación estándar. Apo: apolipoproteína.

${ }^{\dagger} p<0,05 ;{ }^{\dagger \dagger} p<0,01$. Las letras indican los promedios que difieren: ${ }^{a}$ sobrepeso vs normopeso; ${ }^{b}$ obesidad vs. normopeso; ${ }^{\mathrm{c}}$ obesidad abdominal presente vs. obesidad abdominal ausente; ${ }^{d}$ fumador vs. no fumador; e hipertensos vs normotensos.

Prueba de t-student o Análisis de varianza y prueba de Bonferroni como prueba post hoc, según el caso.

pertrigliceridémicos mostraron cifras significativamente más bajas de Apo A-I, mientras que se observó disminución de rHDLc/Apo A-I en sujetos con HDLc baja o LDLc alta.

\section{DISCUSIÓN}

La persistencia de las enfermedades cardiovasculares como la primera causa de mortalidad entre la población venezolana adulta ${ }^{(15)}$ genera la necesidad de estudiar indicadores de riesgo cardiovascular que no se solicitan rutinariamente en los centros públicos de salud de
Venezuela, como es el caso de las apolipoproteínas. Al comparar los valores promedios de las apolipoproteínas estudiadas con los reportados por otros autores, se observa que si bien los niveles de Apo A-I son semejantes a los hallados en Turquía ${ }^{(16)}$ e India ${ }^{(17)}$, los de Apo $B$ son claramente más elevados, lo cual se traduce en una mayor rApo B/Apo A-I. En este estudio, a pesar que las concentraciones promedio de colesterol total y LDLc se encontraron dentro de los rangos deseables, se evidenció un importante porcentaje de individuos que mostraron perfil apolipoprotéico alterado, siendo 27,5\% para Apo A-I baja y alcanzando 45,2 y $60,6 \%$ para Apo B y rApo B/Apo A-I elevadas, respectivamente. Estos

Tabla 4. Niveles Séricos de Apolipoproteínas A-I y B, rApo B/Apo A-I y rHDLc/Apo A-I según patrón de consumo de bebidas alcohólicas*.

\begin{tabular}{|c|c|c|c|c|}
\hline & Apo A-I (mg/dL) & Apo B (mg/dL) & rApo B/Apo A-I & rHDLc/Apo A-I \\
\hline \multicolumn{5}{|l|}{ Consumo de bebidas alcohólicas } \\
\hline No Bebedor (163) & $126,4 \pm 18,0$ & $122,0 \pm 29,1$ & $0,99 \pm 0,31$ & $0,34 \pm 0,08$ \\
\hline Bebedor (58) & $126,6 \pm 20,7$ & $130,0 \pm 28,2$ & $1,08 \pm 0,35$ & $0,34 \pm 0,07$ \\
\hline \multicolumn{5}{|c|}{ Frecuencia de ingesta de bebidas alcohólicas } \\
\hline < 1 día/semana (46) & $126,7 \pm 21,2$ & $132,5 \pm 25,5$ & $1,09 \pm 0,32$ & $0,34 \pm 0,07$ \\
\hline 1-2 día/semana (10) & $123,9 \pm 17,6$ & $121,6 \pm 32,5$ & $1,03 \pm 0,44$ & $0,35 \pm 0,06$ \\
\hline 3 o más días/semana (2) & $139,9 \pm 20,7$ & $115,9 \pm 69,5$ & $0,91 \pm 0,71$ & $0,33 \pm 0,05$ \\
\hline \multicolumn{5}{|c|}{ Cantidad de bebidas alcohólicas ingeridas/día } \\
\hline 1-2 bebidas/día (24) & $128,2 \pm 21,9$ & $140,6 \pm 27,2$ & $1,15 \pm 0,38$ & $0,33 \pm 0,06$ \\
\hline 3 o más bebidas/día (34) & $125,6 \pm 20,2$ & $122,9 \pm 26,9^{\dagger}$ & $1,03 \pm 0,33$ & $0,34 \pm 0,07$ \\
\hline \multicolumn{5}{|c|}{ Frecuencia de ingesta de 6 ó más bebidas alcohólicas/ocasión } \\
\hline Nunca (19) & $128,1 \pm 23,4$ & $135,3 \pm 32,4$ & $1,11 \pm 0,42$ & $0,35 \pm 0,06$ \\
\hline Menos que mensualmente (18) & $125,2 \pm 20,8$ & $136,7 \pm 20,9$ & $1,14 \pm 0,25$ & $0,31 \pm 0,07$ \\
\hline Mensualmente (6) & $134,4 \pm 17,5$ & $119,5 \pm 17,8$ & $0,89 \pm 0,10$ & $0,33 \pm 0,09$ \\
\hline Semanalmente o Diariamente (15) & $123,5 \pm 19,9$ & $119,9 \pm 32,2$ & $1,02 \pm 0,41$ & $0,36 \pm 0,06$ \\
\hline
\end{tabular}

*n entre paréntesis. Resultados expresados como media aritmética desviación estándar. Apo: apolipoproteína.

${ }^{\dagger} p<0,05$, grupo que consumían tres o más bebidas/día vs grupo de 1-2 bebidas/día.

Prueba de t-student o Análisis de varianza y prueba de Bonferroni como prueba post hoc, según el caso. 
Tabla 5. Niveles Séricos de Apolipoproteínas A-I y B, rApo B/Apo A-I y rHDLc/Apo A-I según alteraciones del perfil lipídico*.

\begin{tabular}{|c|c|c|c|c|}
\hline & Apo A-I (mg/dL) & Apo B (mg/dL) & rApo B/Apo A-I & rHDLc/Apo A-I \\
\hline \multicolumn{5}{|l|}{ Colesterol Total } \\
\hline Normal (156) & $128,5 \pm 18,3$ & $114,5 \pm 24,7$ & $0,91 \pm 0,26$ & $0,35 \pm 0,08$ \\
\hline Elevado (65) & $122,1 \pm 19,4^{\text {ta }}$ & $147,7 \pm 25,4^{\dagger \dagger a}$ & $1,26 \pm 0,34^{\dagger+a}$ & $0,33 \pm 0,07$ \\
\hline \multicolumn{5}{|l|}{ HDLc baja } \\
\hline Normal (91) & $130,1 \pm 18,9$ & $116,4 \pm 25,7$ & $0,91 \pm 0,25$ & $0,38 \pm 0,08$ \\
\hline Baja (130) & $124,1 \pm 18,4^{+\mathrm{tb}}$ & $128,9 \pm 30,2^{+\mathrm{tb}}$ & $1,09 \pm 0,36^{++b}$ & $0,32 \pm 0,07^{++b}$ \\
\hline \multicolumn{5}{|l|}{ LDLc } \\
\hline Normal (156) & $127,8 \pm 19,2$ & $115,6 \pm 25,6$ & $0,93 \pm 0,28$ & $0,35 \pm 0,08$ \\
\hline Elevada (65) & $123,8 \pm 17,8$ & $145,0 \pm 26,8^{t \dagger c}$ & $1,21 \pm 0,34^{t+c}$ & $0,32 \pm 0,07^{t+c}$ \\
\hline \multicolumn{5}{|l|}{ Triglicéridos } \\
\hline Normales (124) & $129,8 \pm 17,7$ & $116,5 \pm 26,2$ & $0,92 \pm 0,29$ & $0,35 \pm 0,08$ \\
\hline Elevados (97) & $122,5 \pm 19,5^{\dagger t d}$ & $134,0 \pm 29,8^{t+d}$ & $1,13 \pm 0,34^{\dagger+d}$ & $0,32 \pm 0,08^{t+d}$ \\
\hline
\end{tabular}

"n entre paréntesis. Resultados expresados como media aritmética \pm desviación estándar. Apo: apolipoproteína.

${ }^{\dagger} p<0,05 ;{ }^{+\dagger} p<0,01$. Las letras indican los promedios que difieren: ${ }^{a}$ colesterol total elevado vs normopeso; ${ }^{b} \mathrm{HDL}$ baja vs. HDL normal; ${ }^{\circ}$ LDL elevada vs. LDL normal; d triglicéridos elevados vs. triglicéridos normales.

Prueba de t-student.

resultados podrían ser reflejo de la importante prevalencia de exceso de peso corporal y obesidad abdominal observada, advirtiendo sobre la situación de riesgo cardiovascular de los individuos estudiados, al analizarce en conjunto con datos provenientes de trabajos previos similares ${ }^{(7)}$ y de otros países origen hispano ${ }^{(18)}$.

A diferencia de lo encontrado por Sniwaski et al. ${ }^{(19)}$ en una población saludable argentina, en esta investigación los niveles promedios de las apolipoproteínas no difirieron de acuerdo a la edad aunque la frecuencia de casos con rApo B/Apo A-I elevada en el grupo total al igual que la de Apo B elevada entre los hombres sí se incrementaron con el avance de la edad. Resultados similares se reportaron anteriormente en descendientes del estudio de Framingham ${ }^{(20)}$, lo que se ha explicado a través de una disminución del catabolismo hepático de las LDL con la edad. En dicho trabajo también se informaron niveles mayores de Apo $B$ en los hombres mientras que en el presente estudio las mujeres mostraron una rApo B/Apo A-I promedio significativamente superior, lo cual pudiera justificarse a través de una diferencia de edad que existió a favor de las mujeres en este reporte.

Se demostró un aumento significativo de la concentración de Apo B y de la rApo B/Apo A-I así como disminución de la Apo A-I entre los individuos con exceso de peso según IMC, CC elevada o hipertensos, lo cual coincide con lo demostrado en diversos estudios ${ }^{(19,21)}$. El exceso de tejido adiposo, especialmente del visceral, no solo condiciona la llegada masiva al hígado de ácidos grasos libres e hiperproducción de VLDL que supone una mayor secreción hepática de $A p o B^{(22)}$ sino también un hipercatabolismo de la Apo A-I que es compensado parcialmente por el aumento de su síntesis ${ }^{(23)}$. Por otra parte, es posible que el acúmulo de tejido adiposo visceral también explique la relación observada entre hipertensión y perfil apolipoprotéico alterado, ya que el acúmulo de ese tipo de tejido favorece la activación del sistema renina-angiotensina y del sistema nervioso simpático ${ }^{22)}$. En tal sentido, en este estudio existió una asociación significativa entre hipertensión y CC elevada u obesidad abdominal (datos no mostrados).

Publicaciones anteriores han demostrado la estrecha relación entre habito al tabaco y perfil apolipoprotéico adverso ${ }^{(18,24)}$, hallazgo que se reitera en el presente trabajo. El humo de cigarrillo aumenta la producción de numerosas citoquinas inflamatorias tales como la IL-6 y el factor de necrosis tumoral-alfa ${ }^{(25)}$, las cuales no solo son capaces de incrementar los ácidos grasos libres circulantes sino también inducir insulinoresistencia y síntesis de triglicéridos ${ }^{(26)}$, propiciando la formación de partículas lipoprotéicas que contienen Apo B.

La ingesta de tres o más bebidas/día de vino, licor o cerveza se define como excesiva y asociada a riesgo cardiovascular ${ }^{(27)}$, recomendándose el consumo ligeromoderado de alcohol (1 a 2 bebidas/día) ${ }^{(28)}$. Uno de los mecanismos a través del cual el alcohol ejercería efectos cardioprotectores sería su acción sobre los niveles séricos de las apolipoproteínas, describiéndose niveles significativamente más elevados de Apo A-I y más bajos de Apo B y de rApo B/Apo A-I en individuos con ingesta moderada de alcohol ${ }^{(29,30)}$. En la presente investigación, no se hallaron diferencias en el perfil apolipoprotéico entre los individuos que reportaron ingesta de bebidas alcohólicas y aquellos que no lo hicieron, así como tampoco en relación a la frecuencia de consumo o específicamente a la frecuencia de ingesta de seis o 
más bebidas/ocasión, la cual se considera una embriaguez ocasional no asociada con efectos beneficiosos desde el punto de vista cardiovascular que ocurre especialmente durante viernes y sábado. Es probable que el grupo de participantes que informaron consumo de alcohol fuese muy pequeño para hallar diferencias significativas, sin embargo, contrario a lo que se esperaba, cuando se analizó la cantidad de bebidas ingeridas, se encontró que la concentración promedio de Apo B fue más alta entre los individuos que bebieron la cantidad recomendada (1-2 bebidas/día) con respecto a los que reportaron beber tres o más bebidas/día. Para explicar lo anterior es importante considerar la posibilidad que un subreporte del consumo de alcohol en la muestra estudiada haya influido sobre los resultados obtenidos pues datos oficiales demuestran que el alcohol es la droga más consumida entre los venezolanos, ingiriendo frecuentemente altas dosis de alcohol (diez unidades de cerveza y de tres a cuatro tragos para los licores fuertes como el ron, whisky y vodka) sin grandes diferencias por sexo y a menudo durante los fines de semana (31). En ese orden de ideas, es probable que los individuos categorizados como bebedores de alcohol en la cantidad recomendada sostuvieran en realidad ingestas mucho más elevadas que conducen a un perfil lipoprotéico desfavorable. Estudios de diseño prospectivo podrían aclarar la relación entre las apolipoproteínas estudiadas y el consumo de alcohol en la población venezolana.

Diferentes investigaciones realizadas fuera de Venezuela han encontrado fuerte correlación entre los niveles los lípidos plasmáticos y las concentraciones de Apo B y Apo A-I ${ }^{(9,16,21)}$. Por su parte, un estudio de una población joven de la ciudad de Maracaibo también demostró una elevada correlación entre Apo B y LDL-c o colesterol total ${ }^{(32)}$. Tales evidencias coinciden con los datos obtenidos en la presente investigación donde se observó una disminución significativa a Apo A-I y aumento de la Apo $B$ y rApo B/Apo A-I entre los individuos con hipercolesterolemia, hipertrigliceridemia o HDLc baja, resultados que se esperaban dado que la Apo $B$ constituye un resumen de todas las partículas aterogénicas Apo $B$ dependientes mientras que la Apo A-I refleja la vía ateroprotectora del metabolismo lipídico al participar activamente en la depuración del colesterol de los tejidos.

La reducción de la rHDLc/Apo A-I se interpreta como un descenso de la eficiencia del transporte reverso del colesterol (TRC) desde los tejidos extrahepáticos, lo cual se refleja en la circulación con partículas de HDL pobres en colesterol esterificado ${ }^{(5)}$. En el presente estudio, la rHDLc/Apo A-I disminuyó significativamente en los individuos con 56 o más años de edad, hallazgo reportado por Berrougui et al. (33) quienes han demostrado, mediante técnicas sofisticadas para medir TRC, disminución de la capacidad de las HDL de los individuos mayores para promover dicho transporte. De igual manera, los participantes fumadores o con hipertrigliceridemia mostraron una rHDLc/Apo A-I promedio significativamente más baja que aquellos no fumadores o normotrigliceridémicos. El hábito tabáquico se asocia con deterioro del remodelamiento de la HDL por inhibición de varias de las etapas del TRC ${ }^{(34)}$, mientras que el aumento de los triglicéridos se acompaña de disminución del tamaño de las partículas de HDL, por fallas en la maduración de esta lipoproteína y con ello de la eficacia del TRC ${ }^{(35)}$

De acuerdo con la revisión bibliográfica realizada, no se disponen de trabajos anteriores que hayan comparado la rHDLc/Apo A-I según IMC, CC o presión arterial elevada. Aunque se ha descrito en obesos déficit de pre- $\beta-H D L$, una subclase de HDL estrechamente asociada al TRC ${ }^{(36)}$, en este trabajo la rHDLc/Apo A-I no mostró cambios significativos entre los participantes con exceso de peso ni entre los hipertensos, en cuyo caso se esperaba por la estrecha asociación, que se comentó anteriormente, entre hipertensión y CC elevada. Situación similar ocurrió al no encontrarse diferencias significativas cuando se comparó la rHDLc/Apo A-I según el patrón de consumo de alcohol, ya que se ha probado que el consumo moderado de alcohol estimula el TRC (37). No obstante, se requieren estudios adicionales que esclarezcan la verdadera capacidad de la rHDLc/Apo A-I para reflejar el TRC en obesos, hipertensos y consumidores de alcohol.

Por último, en razón del diseño transversal del trabajo y del muestreo no probabilístico aplicado, es preciso señalar que la prevalencia de perfil apolipoprotéico alterado informada en esta investigación puede corresponder a una situación estrictamente local o del ámbito institucional en el cual se desarrolló la recolección de la muestra estudiada, por lo que los datos presentados no pueden ser extrapolados a la comunidad o a la población venezolana ni pueden servir para inferir relaciones causales.

En conclusión, se demostró en los individuos estudiados, alta prevalencia de perfil apolipoprotéico alterado, lo cual se asoció con los principales factores de riesgo cardiovascular. La rHDLc/Apo A-I disminuyó con la edad, hábito tabáquico y aumento de LDLc y triglicéridos. Se recomienda abordar en estudios más amplios, el efecto del consumo de alcohol sobre el perfil apolipoprotéico en población venezolana. Los resultados de esta investigación apoyarían la inclusión de la determinación de los niveles séricos de las apolipoproteínas estudiadas como parte de la valoración del metabolismo lipídico en los centros de atención de salud venezolanos. 


\section{Contribuciones de Autoría}

NR participó en la concepción y diseño del trabajo, recolección y obtención de los resultados, análisis e interpretación de los datos, redacción del manuscrito y aportó material de estudio. VC y FC participaron en el diseño del trabajo, recolección, obtención de los resultados y análisis de los datos así como en la redacción de la primera versión del manuscrito. ME participó en la recolección y obtención de los resultados y aportó material de estudio. UL aportó pacientes y material de estudio. JCG aportó material de estudio. Todos los autores revisaron en forma crítica las versiones preliminares del manuscrito y aprobaron la versión final del trabajo.

\section{Fuentes de Financiamiento}

Autofinanciado.

\section{Conflictos de Interés}

Los autores declaran no tener conflicto de interés en la publicación del presente artículo.

\section{REFERENCIAS BIBLIOGRÁFICAS}

1. Davidson MH. Apolipoprotein measurements: is more widespread use clinically indicated?. Clin Cardiol. 2009;32(9):482-6.

2. Marcovina S, Packard CJ. Measurement and meaning of apolipoprotein A1 and apolipoprotein B plasma levels. J Intern Med. 2006;259(5):437-46.

3. McQueen MJ, Hawken S, Wang X, Ounpuu S, Sniderman A, Probstfield J, et. al. Lipids, lipoproteins, and apolipoproteins as risk markers of myocardial infarction in 52 countries (the INTERHEART study): a case-control study; INTERHEART study investigators. Lancet. 2008;372(9634):224-33.

4. Sierra-Johnson J, Fisher RM, Romero-Corral A, Somers VK, Lopez-Jimenez F, Öhrvik J, et. al. Concentration of apolipoprotein B is comparable with the apolipoprotein B/ apolipoprotein A-I ratio and better than routine clinical lipid measurements in predicting coronary heart disease mortality: findings from a multi-ethnic US population. Eur Heart J. 2009;30(6):710-7.

5. Espondaburu OR. Hipertrigliceridemia: influencia sobre parámetros que estiman el transporte reverso del colesterol. Acta Bioquím Clín Latinoam. 2006;40(2):165-72.

6. Florez H, Silva E, Fernández V, Ryder E, Sulbarán T, Campos G, et. al. Prevalence and risk factors associated with the metabolic syndrome and dyslipidemia in White, Black, Amerindian and Mixed Hispanics in Zulia State, Venezuela. Diabetes Res Clin Pract. 2005;69(1):63-77.

7. Ruiz-Fernández N, Espinoza M, Barrios $E$, Reigosa $A$. Cardiometabolic factors in a comunity located at Valencia city, Venezuela. Rev Salud Publica (Bogota). 2009;11(3): 383-94.

8. Becerra V, Torres A, Arata G, Velázquez E. Prevalencia del síndrome metabólico en la población urbana de Mucuchíes, Mérida Venezuela. Rev Venez Endocrinol Metabol. 2009;7(3):16-22.
9. Riediger ND, Bruce SG, Young TK. Cardiovascular risk according to plasma apolipoprotein and lipid profiles in a Canadian First Nation. Prev Chronic Dis. 2011;8(1):A05.

10. Rodríguez-Larralde A, Mijares ME, Nagy E, Espinosa R, Ryder E, Diez-Ewald MP, et. al. Relación entre el nivel socioeconómico y hábitos de vida, con el fibrinógeno y el factor Von Willebrand en venezolanos sanos y con cardiopatía isquémica. Invest Clin. 2005;46(2):157-68.

11. World Health Organization. Physical Status: The Use and Interpretation of Anthropometry. Report of a WHO Expert Committee. WHO Technical Report Series 854. Geneva: World Health Organization; 1995.

12. Joint National Committee on Prevention, Detection, Evaluation, and Treatment of High Blood Pressure. The seventh report of the joint national committee on prevention, detection, evaluation, and treatment of high blood pressure: the JNC 7 report. JAMA. 2003;289(19):2560-71.

13. National Cholesterol Education Program (NCEP) Expert Panel on Detection, Evaluation, and Treatment of High Blood Cholesterol in Adults (Adult Treatment Panel III). Third report of the National Cholesterol Education Program (NCEP) Expert Panel on Detection, Evaluation, and Treatment of High Blood Cholesterol in Adults (Adult Treatment Panel III). Final Report. Circulation. 2002;106(25):3143-421.

14. Babor TF, Higgins- Biddle JC, Saunders JB, Monteiro MG. AUDIT, The Alcohol Use Disorders Identification Test. $2^{\mathrm{a}}$ ed. Geneva: World Health Organization; 2001.

15. Ministerio del Poder Popular para la Salud de la República Bolivariana de Venezuela. Anuario de Mortalidad 2007. Caracas: MPPS de Venezuela; 2009.

16. Onat A. Lipids, lipoproteins and apolipoproteins among turks, and impact on coronary heart disease. Anadolu Kardiyol Derg. 2004;4(3):236-45.

17. Ashavaid TF, Kondkar AA, Todur SP, Dherai AJ, Morey J, Raghavan R. Lipid, lipoprotein, apolipoprotein and lipoprotein(a) levels: reference intervals in a healthy Indian population. J Atheroscler Thromb. 2005;12(5):251-9.

18. Montero JC. Epidemiología de la obesidad en siete países de América Latina. Form Contin Nutr Obes. 2002;5(6):325-30.

19. Siniawski D, Masson W, Bluro I, Sorroche P,Sscordo W, Krauss J, et. al. Niveles plasmáticos de apolipoproteínas en una población saludable de la Argentina: implicaciones en prevención cardiovascular. Rev Argent Cardiol. 2010;78(2):123-8.

20. Schaefer EJ, Lamon-Fava S, Cohn SD, Schaefer MM, Ordovas JM, Castelli WP, et. al. Effects of age, gender, and menopausal status on plasma low density lipoprotein cholesterol and apolipoprotein B levels in the Framingham Offspring Study. J Lipid Res.1994;35(5):779-92.

21. Krittayaphong R, Chotinaiwatarakul $C$, Kangkagate $C$, Bhuripanyo K, Mahanonda N. The association of apolipoprotein B and low density lipoprotein with cardiovascular risk factors in the Thai population. J Med Assoc Thai. 2006;89 Suppl 5:S1-7

22. Mathieu $P$, Poirier $P$, Pibarot $P$, Lemieux I, Després JP. Visceral obesity: the link among inflammation, hypertension, and cardiovascular disease. Hypertension. 2009;53(4):577-84.

23. Welty FK, Lichtenstein AH, Lamon-Fava S, Schaefer EJ, Marsh JB. Effect of body mass index on apolipoprotein A-I 
kinetics in middle-aged men and postmenopausal women. Metabolism. 2007;56(7):910-4.

24. Yasue H, Hirai N, Mizuno Y, Harada E, Itoh T, Yoshimura $\mathbf{M}$, et. al. Low-Grade inflammation, thrombogenicity, and atherogenic lipid profile in cigarette smokers. Circ J. 2006;70(1):8-13.

25. Arnson Y, Shoenfeld Y, Amital H. Effects of tobacco smoke on immunity, inflammation and autoimmunity. J Autoimmun. 2010;34(3):J258-65.

26. Sánchez-Muñoz F, García-Macedo R, Alarcón-Aguilar F, Cruz M. Adipocinas, tejido adiposo y su relación con células del sistema inmune. Gac Méd Méx. 2005;141(6):50512

27. Kloner RA, Rezkalla SH. To Drink or not to Drink? That is the Question. Circulation. 2007;116(11):1306-17.

28. US Department of Agriculture and US Department of Health and Human Services. Dietary Guidelines for Americans, Alcoholic Beverages. Washington, DC: Government Printing Office; 2005.

29. Nanchahal K, David Ashton W, Wood DA. Alcohol consumption, metabolic cardiovascular risk factors and hypertension in women. Int J Epidemiol. 2000;29(1):57-64.

30. Simonsson M, Schmidt C, Sigurdadottir V, Helenius ML, Fagerberg B. Life style habits such as alcohol consumption and physical activity in relation to serum apoB /apoA-I ratio amongst 64 -year-old women with varying degrees of glucose tolerance. J Intern Med. 2007;262(5):537-44.

31. Oficina Nacional Antidrogas. Primera Encuesta Epidemiológica a Hogares Sobre Consumo de Drogas [Internet]. Caracas: Oficina Nacional Antidrogas; 2005 [citado el 15 de enero de 2011]. Disponible en: http://www.ona.gob.ve/Pdf/ Encuesta_Epidemiologica_2005.pdf
32. Bonezzi SE, Casanova RI, Gómez GM, Casanova A, Ortega Al. Niveles de apolipoproteína-B y LDL-colesterol en una población joven de la ciudad de Maracaibo. Medicina (Ribeirão Preto). 2002;35(4):470-7.

33. Berrougui H, Isabelle M, Cloutier M, Grenier G, Khalil A. Age-related impairment of HDL-mediated cholesterol efflux. J Lipid Res. 2007;48(2):328-36.

34. Zaratin AC, Quintão EC, Sposito AC, Nunes VS, Lottenberg AM, Morton RE, et. al. Smoking prevents the intravascular remodeling of high-density lipoprotein particles: implications for reverse cholesterol transport. Metabolism. 2004;53(7):858-62.

35. Tian L, Xu Y, Fu M, Peng T, Liu Y, Long S. The impact of plasma triglyceride and apolipoproteins concentrations on high-density lipoprotein subclasses distribution. Lipids Health Dis. 2011;10:17.

36. Rashid S, Genest J. Effect of obesity on high-density lipoprotein metabolism. Obesity (Silver Spring). 2007;15(12):2875-88.

37. Sierksma A, Vermunt SH, Lankhuizen IM, van der Gaag MS, Scheek LM, Grobbee DE, et. al. Effect of moderate alcohol consumption on parameters of reverse cholesterol transport in postmenopausal women. Alcohol Clin Exp Res. 2004;28(4):662-6.

Correspondencia: Nelina Ruíz Fernández.

Dirección: Calle Acuario, N. ${ }^{\circ} 88-20$, Urb. Trigal Norte, Valencia, Estado. Carabobo, Venezuela.

Teléfono: (58) 241 842-6674

Correo electrónico: nelinaruiz@yahoo.com

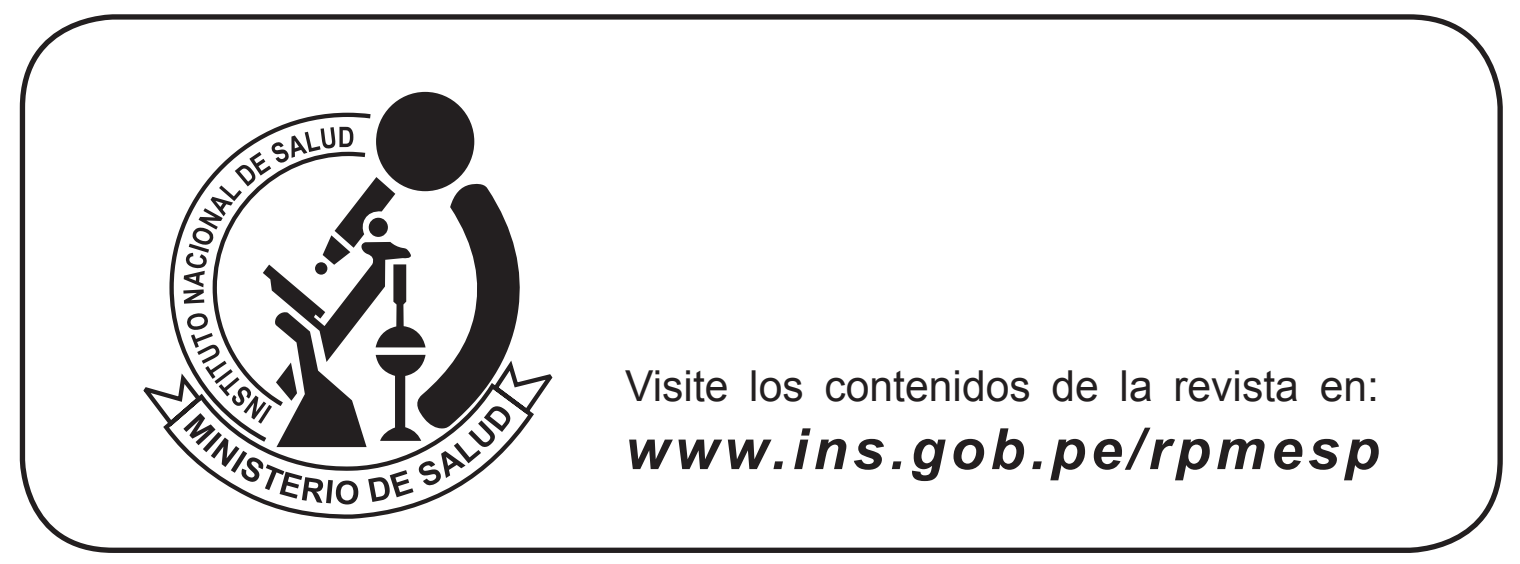

